\title{
乌ु
}

\section{QCD Phase Transition with Chiral Quarks and Physical Quark Masses}

\author{
Tanmoy Bhattacharya, ${ }^{1}$ Michael I. Buchoff, ${ }^{2,3}$ Norman H. Christ, ${ }^{4,}$ H.-T. Ding, ${ }^{5}$ Rajan Gupta, ${ }^{1}$ Chulwoo Jung, ${ }^{6}$ \\ F. Karsch ${ }^{6,7}$ Zhongjie Lin, ${ }^{4}$ R. D. Mawhinney, ${ }^{4}$ Greg McGlynn, ${ }^{4}$ Swagato Mukherjee, ${ }^{6}$ David Murphy, ${ }^{4}$ \\ P. Petreczky, ${ }^{6}$ Dwight Renfrew, ${ }^{4}$ Chris Schroeder, ${ }^{2}$ R. A. Soltz, ${ }^{2}$ P. M. Vranas, ${ }^{2}$ and Hantao Yin $^{4}$
}

(HotQCD Collaboration)

\author{
${ }^{1}$ Theoretical Division, Los Alamos National Laboratory, Los Alamos, New Mexico 87544, USA \\ ${ }^{2}$ Physics Division, Lawrence Livermore National Laboratory, Livermore, California 94550, USA \\ ${ }^{3}$ Institute for Nuclear Theory, P.O. Box 351550, Seattle, Washington 98195-1550, USA \\ ${ }^{4}$ Physics Department, Columbia University, New York, New York 10027, USA \\ ${ }^{5}$ Key Laboratory of Quark and Lepton Physics (MOE) and Institute of Particle Physics, \\ Central China Normal University, Wuhan 430079, China \\ ${ }^{6}$ Physics Department, Brookhaven National Laboratory, Upton, New York 11973, USA \\ ${ }^{7}$ Fakultät für Physik, Universität Bielefeld, D-33615 Bielefeld, Germany
}

(Received 17 March 2014; published 18 August 2014)

\begin{abstract}
We report on the first lattice calculation of the QCD phase transition using chiral fermions with physical quark masses. This calculation uses $2+1$ quark flavors, spatial volumes between $(4 \mathrm{fm})^{3}$ and $(11 \mathrm{fm})^{3}$ and temperatures between 139 and $196 \mathrm{MeV}$. Each temperature is calculated at a single lattice spacing corresponding to a temporal Euclidean extent of $N_{t}=8$. The disconnected chiral susceptibility, $\chi_{\text {disc }}$ shows a pronounced peak whose position and height depend sensitively on the quark mass. We find no metastability near the peak and a peak height which does not change when a $5 \mathrm{fm}$ spatial extent is increased to $10 \mathrm{fm}$. Each result is strong evidence that the QCD "phase transition" is not first order but a continuous crossover for $m_{\pi}=135 \mathrm{MeV}$. The peak location determines a pseudocritical temperature $T_{c}=155(1)(8) \mathrm{MeV}$, in agreement with earlier staggered fermion results. However, the peak height is $50 \%$ greater than that suggested by previous staggered results. Chiral $S U(2)_{L} \times S U(2)_{R}$ symmetry is fully restored above $164 \mathrm{MeV}$, but anomalous $U(1)_{A}$ symmetry breaking is nonzero above $T_{c}$ and vanishes as $T$ is increased to $196 \mathrm{MeV}$.
\end{abstract}

DOI: 10.1103/PhysRevLett.113.082001

PACS numbers: $12.38 . \mathrm{Gc}, 11.15 . \mathrm{Ha}$

As the temperature of the $\mathrm{QCD}$ vacuum is increased above the QCD energy scale $\Lambda_{\mathrm{QCD}}=300 \mathrm{MeV}$, asymptotic freedom implies that the vacuum breaking of chiral symmetry must disappear and the familiar chirally asymmetric world of massive nucleons and light pseudo-Goldstone bosons must be replaced by an $S U(2)_{L} \times S U(2)_{R}$ symmetric plasma of nearly massless up and down quarks and gluons. Predicting, observing, and characterizing this transition has been an experimental and theoretical goal since the 1980s. General principles are consistent with this being either a first-order transition for sufficiently light pion mass or a second-order transition in the $O(4)$ universality class at zero pion mass with crossover behavior for nonzero $m_{\pi}$. While second order behavior is commonly expected, firstorder behavior may be more likely if anomalous $U(1)_{A}$ symmetry is partially restored at $T_{C}$ resulting in an effective $U_{L}(2) \times U_{R}(2)$ symmetry $[1,2]$.

The importance of the $S U(2)_{L} \times S U(2)_{R}$ chiral symmetry of QCD for the phase transition has motivated the widespread use of staggered fermions in lattice studies of QCD thermodynamics because this formulation possesses one exact chiral symmetry at finite lattice spacing, broken only by the quark mass. However, the flavor symmetry of the staggered fermion formulation is complicated showing an $S U_{L}(4) \times S U_{R}(4)$ "taste" symmetry that is broken by lattice artifacts and made to resemble the physical $S U(2)_{L} \times$ $S U(2)_{R}$ symmetry by taking the square root of the Dirac determinant, a procedure believed to have a correct but subtle continuum limit for nonzero quark masses.

Because of these limitations, it is important to study these phenomena using a different fermion formulation, ideally one which supports the full $S U(2)_{L} \times S U(2)_{R}$ chiral symmetry of QCD at finite lattice spacing. It is such a study which we report here. We use Möbius domain wall fermions [3], a formulation in which the fermions are defined on a five-dimensional lattice. While the extent in the fifth dimension, $L_{s}=16$ or 24 , makes the calculation 16 to 24 times more costly, the resulting theory possesses an accurate $S U(2)_{L} \times S U(2)_{R}$ symmetry, broken only by the input quark mass and the highly suppressed mixing between the left and right four-dimensional boundaries, where the low-energy fermions propagate. This residual 
chiral asymmetry is a short-distance phenomenon whose leading long-distance effect is to add a constant $m_{\text {res }}$ to each input quark mass, $m_{q}$, giving a total mass $\tilde{m}_{q}=m_{q}+m_{\text {res }}$. Here $m_{\text {res }} \approx 3 \mathrm{MeV}$ and further residual chiral symmetry breaking is $O\left(m_{\mathrm{res}} a^{2}\right)$ [4].

Because of this computational cost, the calculation reported here uses only one lattice spacing, $a$, at each temperature, corresponding to a temporal extent of $N_{t}=8$. The good agreement with experiment for $f_{\pi}$ and $f_{K}$ computed at our largest lattice spacing and a comparison of zero temperature results at our $T \approx 170$ lattice spacing with those from two smaller lattice spacings [5], suggest $\approx 5 \%$ discretization errors in our results. In contrast, the less costly staggered fermion calculations use $N_{t}=8,10,12$, and 16. However, to make a controlled continuum extrapolation, the staggered fermion discretization errors are assumed to behave as $a^{2}$. Potential nonlinearities in the taste-breaking effects, which in zero-temperature staggered fermion calculations are handled using staggered chiral perturbation theory, are ignored because of the absence of a corresponding theory of finite-temperature taste breaking.

Methods. - The present calculation with $m_{\pi}=135 \mathrm{MeV}$ and $32^{3} \times 8$ and $64^{3} \times 8$ volumes extends earlier domain wall results with $m_{\pi}=200 \mathrm{MeV}$ and $16^{3} \times 8,24^{3} \times 8$ and $32^{3} \times 8$ volumes [6-8]. We use the same combination of Iwasaki gauge action and dislocation suppressing determinant ratio (DSDR) exploited to reduce residual chiral symmetry breaking in this earlier work. To enable calculations at $m_{\pi}=135 \mathrm{MeV}$ with available computing resources we have changed the Shamir domain wall formulation to Möbius [3]. By choosing the Möbius parameters $b$ and $c$ of Ref. [3] so that $b-c=1$, we insure that our Möbius Green's functions will agree at the $0.1 \%$ level with those of Shamir evaluated at a much larger $L_{s}$. Thus, except for their quark masses, our $m_{\pi}=200$ and $135 \mathrm{MeV}$ calculations are equivalent, including lattice artifacts.

Table I lists the parameters for the $m_{\pi}=135 \mathrm{MeV}$ ensembles and results for the residual mass. At the lowest temperatures, more than $90 \%$ of the quark mass is generated by residual chiral symmetry breaking. In addition to these 13 ensembles with $N_{t}=8$, two calculations were performed at $T=0$ with space-time volume $32^{3} \times 64$. These used $\beta=1.633$ (first reported here) and $\beta=1.75$ [5], corresponding to $T=139 \mathrm{MeV}$ and $T \approx 170 \mathrm{MeV}$ when $N_{t}=8$.

The choices of quark masses and assigned temperatures given in Table I were estimated from earlier work $[5,6]$. Results from the new zero temperature ensemble at $\beta=1.633$, obtained with the quark masses shown in Table I, are summarized in Table II and provide a check of these estimates. The resulting lattice spacing and pion mass are close to our targets while the kaon mass is lighter than expected, which may be unimportant for the quantities studied here. Of special interest is a comparison of the residual mass for this value of $\beta$ given in Tables I and II.
TABLE I. A summary of the $m_{\pi}=135 \mathrm{MeV}$ ensembles. The units are $\mathrm{MeV}$ for the temperature $T$ and $10^{-5} / a$ for the masses $m_{l}, m_{s}$, and $m_{\text {res }} . N_{\text {st }}, N_{\text {tot }}$, and $N_{\sigma}$ label the number of independent streams, the total equilibrated time units, and the number of sites in each spatial direction, respectively.

\begin{tabular}{lccccccccr}
\hline \hline$T$ & $\beta$ & $N_{\sigma}$ & $L_{s}$ & $c$ & $m_{l}$ & $m_{s}$ & $m_{\text {res }}$ & $N_{\text {st }}$ & $N_{\text {tot }}$ \\
\hline 139 & 1.633 & 32 & 24 & 1.5 & 22 & 5960 & $219(1)$ & 4 & 5768 \\
139 & 1.633 & 64 & 24 & 1.5 & 22 & 5960 & $219(1)$ & 1 & 380 \\
149 & 1.671 & 32 & 16 & 1.5 & 34 & 5538 & $175(1)$ & 4 & 7823 \\
149 & 1.671 & 64 & 16 & 1.5 & 34 & 5538 & $175(1)$ & 3 & 2853 \\
154 & 1.689 & 32 & 16 & 1.5 & 75 & 5376 & $120(4)$ & 4 & 6108 \\
159 & 1.707 & 32 & 16 & 1.5 & 112 & 5230 & $91(1)$ & 3 & 8714 \\
159 & 1.707 & 64 & 16 & 1.5 & 112 & 5230 & $91(1)$ & 2 & 3431 \\
164 & 1.725 & 32 & 16 & 1.5 & 120 & 5045 & $68(5)$ & 4 & 7149 \\
168 & 1.740 & 32 & 16 & 1.2 & 126 & 4907 & $57(1)$ & 2 & 5840 \\
168 & 1.740 & 64 & 16 & 1.2 & 126 & 4907 & $57(1)$ & 1 & 1200 \\
177 & 1.771 & 32 & 16 & 1.0 & 132 & 4614 & $43(1)$ & 2 & 8467 \\
186 & 1.801 & 32 & 16 & 1.0 & 133 & 4345 & $26(1)$ & 2 & 10127 \\
195 & 1.829 & 32 & 16 & 0.9 & 131 & 4122 & $19(1)$ & 2 & 10124 \\
\hline \hline
\end{tabular}

The $1.1 \%$ discrepancy is a measure of discretization error. Likewise, the comparison with experiment of $f_{\pi}$ and $f_{K}$ gives $6 \%$ and $4 \%$ errors, indicating the size of discretization effects.

Results.-Our most dramatic result is the temperaturedependent, disconnected chiral susceptibility $\chi_{\text {disc }}$, plotted in Fig. 1. Three of the four lower curves show earlier results with $m_{\pi}=200 \mathrm{MeV}$ on $16^{3}, 24^{3}$, and $32^{3}$ volumes. A significant decrease in $\chi_{\text {disc }}$ is seen for temperatures below $165 \mathrm{MeV}$ as the volume is increased above $16^{3}$, a volume dependence anticipated in earlier scaling [9-11] and model [12] studies. The two higher curves show a large increase in $\chi_{\text {disc }}$ in the entire transition region for $m_{\pi}=135 \mathrm{MeV}$ and both $32^{3}$ and $64^{3}$ volumes. The ratio of peak heights for the $m_{\pi}=135$ and $200 \mathrm{MeV}, 32^{3}$ data is 2.1(0.2), which is consistent with the ratio 1.86 predicted by universal $O(4)$ scaling $\sim \tilde{m}_{l}^{1 / \delta-1} \propto m_{\pi}^{-1.5854}$, only if the regular, massindependent part of $\chi_{\text {disc }}$ is small.

This comparison of $\chi_{\text {disc }}$ with $O(4)$ scaling neglects the connected part of the chiral susceptibility. In fact, the connected chiral susceptibility depends mildly on

TABLE II. Results at $\beta=1.633$ and $T=0$ (in lattice units and $\mathrm{MeV}$ ) from 25 configurations separated by at least 20 time units. We use $M_{\Omega}$ to fix the scale. Also listed are the experimental values.

\begin{tabular}{lccc}
\hline \hline & $1 / a$ & $\mathrm{MeV}$ & Expt.(MeV) \\
\hline$m_{\pi}$ & $0.1181(5)$ & $129.2(5)$ & 135 \\
$m_{K}$ & $0.4230(5)$ & $462.5(5)$ & 495 \\
$m_{\Omega}$ & $1.530(3)$ & 1672.45 & 1672.45 \\
$T=1 / 8 a$ & 0.125 & $136.7(3)$ & $\ldots$ \\
$f_{\pi}$ & $0.1263(2)$ & $138.1(2)$ & 130.4 \\
$f_{K}$ & $0.1483(4)$ & $162.2(4)$ & 156.1 \\
$m_{\text {res }}$ & $0.00217(2)$ & $\ldots$ & $\cdots$ \\
\hline \hline
\end{tabular}




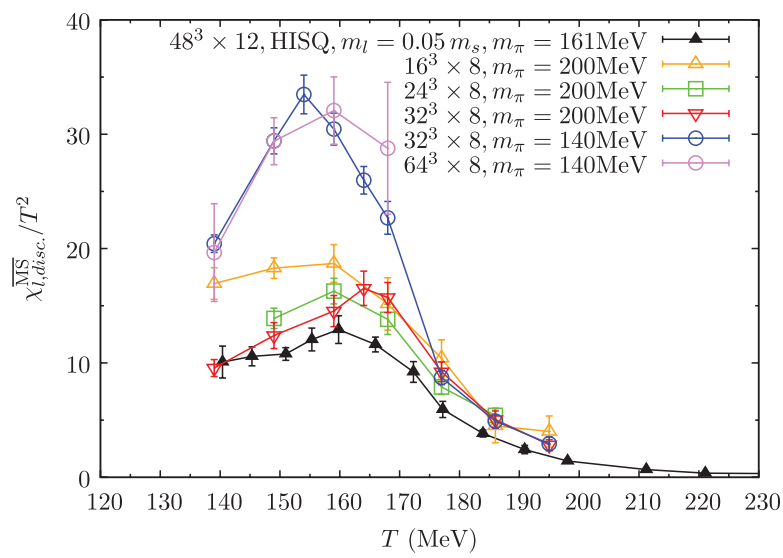

FIG. 1 (color online). The dependence of the disconnected chiral susceptibility on $T$ for $m_{\pi}=135$ and $200 \mathrm{MeV}$. The $m_{\pi}=135 \mathrm{MeV}$ data show a near $2 \times$ increase over that for $m_{\pi}=200 \mathrm{MeV}$. HISQ results for $m_{\pi}=161 \mathrm{MeV}[7,13]$ are also plotted. The errors in the conversion of $\chi_{l \text {,disc }}$ from bare to $\overline{\mathrm{MS}}$ values, common to all of the DWF results, are not shown.

temperature and quark mass (expected if the $\delta$ screening mass remains nonzero at $T_{c}$ ) and so does not contribute to the singular part of the chiral susceptibility.

Also shown in this figure are highly improved staggered quarks (HISQ) results for $N_{t}=12$ and a Goldstone pion mass of $161 \mathrm{MeV}$ [7,13]. If scaled to $m_{\pi}=135 \mathrm{MeV}$ assuming this same $m_{\pi}^{-1.5854}$ behavior, the HISQ value for $\chi_{\text {disc }}$ is $50 \%$ smaller than that seen here. This discrepancy reaffirms the importance of an independent study of the order of the transition and calculation of $T_{c}$ using chiral quarks. (Note in this DWF-HISQ comparison only the ratios of lattice quark and pion masses and lattice scales are needed. The perturbative uncertainties in connecting to the $\overline{\mathrm{MS}}$ scheme cancel.)

The peak shown in Fig. 1 implies a pseudocritical temperature of $155(1)(8) \mathrm{MeV}$. The central value and statistical error are obtained by fitting the $T=149,154$, and $159 \mathrm{MeV}$ values of $\chi_{\text {disc }}^{\overline{\mathrm{MS}}}$ to a parabola. The second, systematic error reflects the expected 5\% discretization error. We do not include a finite-volume systematic error. While typically neglected when $N_{\sigma} / N_{t} \geq 4$, we lack the data needed for an empirical estimate. This result for $T_{c}$ is consistent with the staggered-fermion continuum limit $[13,14]$.

The order of the QCD phase transition can be studied using the time history of the chiral condensate for $T \approx T_{c}$. Figure 2 shows four time histories of $\left\langle\bar{q}_{l} q_{l}\right\rangle$ at $T=154 \mathrm{MeV}$. All four streams fluctuate over the same range, showing no metastable behavior and no difference between streams starting from ordered or disordered configurations. This and the failure of $\chi_{\text {disc }}$ to grow when the volume is increased from $32^{3}$ to $64^{3}$ (a contribution to $\chi_{\text {disc }}$ from tunneling between two metastable states should have increased by $2^{3}$ ) provide strong evidence that for $m_{\pi}=135 \mathrm{MeV}$, the QCD transition is not first order but a crossover, a conclusion consistent with previous staggered work [13,15-17].

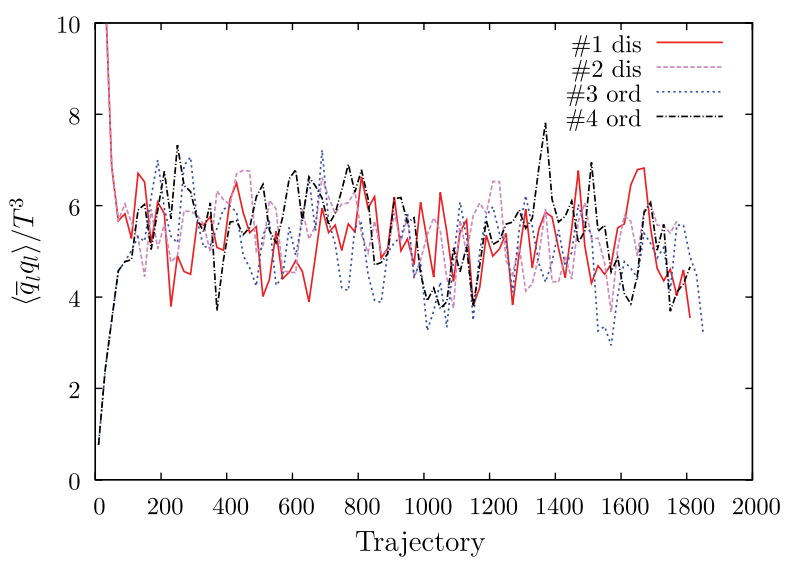

FIG. 2 (color online). The time histories of $\left\langle\bar{q}_{l} q_{l}\right\rangle$ for four streams at $T=154 \mathrm{MeV}$. Streams beginning with an ordered or disordered configuration are labeled ord or dis. Each point averages results from 10 random sources on 20 configurations, separated by one time unit.

In Fig. 3 we show the $S U(2)_{L} \times S U(2)_{R}$-breaking differences between the susceptibilities $\chi_{\pi}$ and $\chi_{\sigma}$ and between $\chi_{\delta}$ and $\chi_{\eta}$. Each pair of fields, $(\vec{\pi}, \sigma)$ and $(\vec{\delta}, \eta)$ forms a four-dimensional representation of $S U(2)_{L} \times S U(2)_{R}$. These $S U(2)_{L} \times S U(2)_{R}$-breaking differences are large below $T_{c}$ but have become zero for $T>164 \mathrm{MeV}$. In Fig. 4 we show the difference $\chi_{\pi}-\chi_{\delta}$. These quantities are related by the anomalous $U(1)_{A}$ transformation, a symmetry of the classical theory that is broken by the axial anomaly. Figure 4 shows that this symmetry is not restored until $T \geq 196 \mathrm{MeV}$. Also shown is the result from our earlier $m_{\pi}=200 \mathrm{MeV}$ calculation [7]. The expected increase in $\chi_{\pi}-\chi_{\delta}$ with decreasing pion mass is seen for $T \leq T_{c}$. Above $T=168 \mathrm{MeV}$ this difference has become mass independent, confirming our earlier conclusion that this

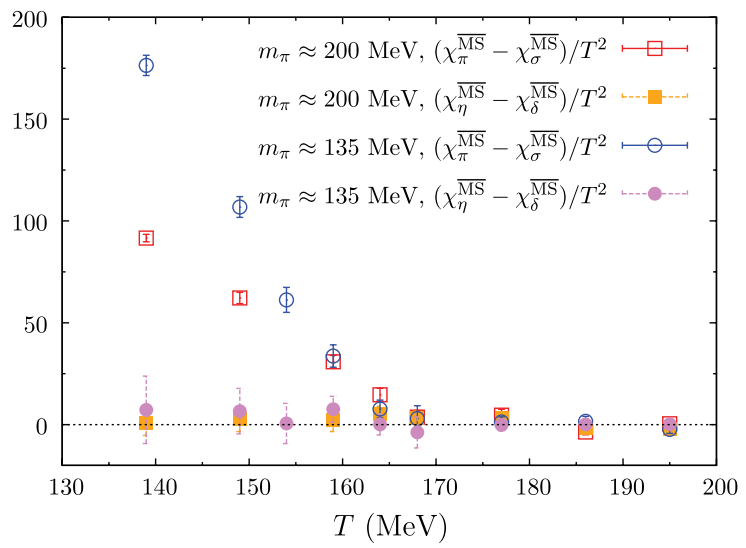

FIG. 3 (color online). Two susceptibility differences are shown that reflect the $S U(2)_{L} \times S U(2)_{R}$ symmetry of QCD and our chiral fermion formulation. Below $T_{c}$ this symmetry is spontaneously broken. For $T>164 \mathrm{MeV}$ we see accurate chiral symmetry. Here and in Fig. 4 only $32^{3}$ data is shown. Little volume dependence is seen for these differences [7] for $16^{3}, 24^{3}$, and $32^{3}$ volumes and $m_{\pi}=200 \mathrm{MeV}$. 


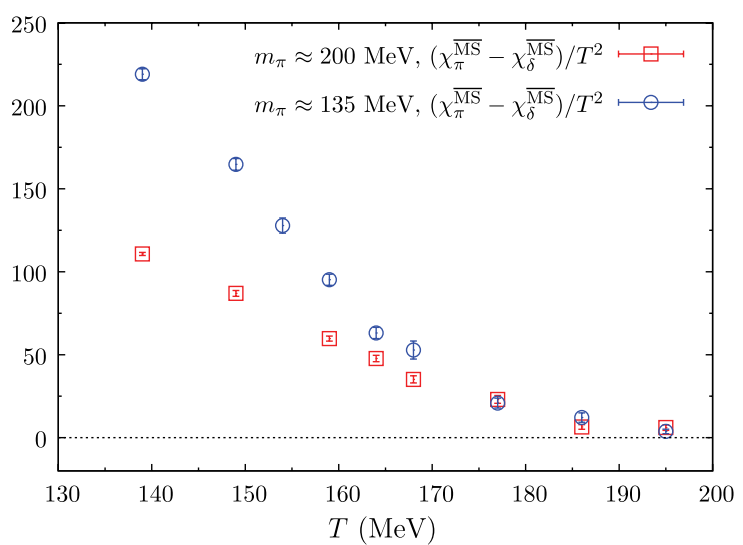

FIG. 4 (color online). The $T$ dependence of the anomalous $U(1)_{A}$-breaking difference $\chi_{\pi}-\chi_{\delta}$, which remains nonzero and becomes mass independent for $T>168 \mathrm{MeV}$.

nonzero value results from the axial anomaly, not the small quark mass.

Conclusion.-We have presented results from the first study of the QCD phase transition using chirally symmetric lattice fermions, physical quark masses, and therefore three degenerate pions with $m_{\pi} \approx 135 \mathrm{MeV}$. We find $T_{c}=155(1)(8) \mathrm{MeV}$, similar to previous staggered fermion results, and see crossover behavior, consistent with a second order critical point at zero quark mass. We show that anomalous symmetry breaking extends to temperatures $\approx 30 \mathrm{MeV}$ above $T_{c}$. Finally, we see a factor of 2 increase in the disconnected chiral susceptibility, $\chi_{\text {disc }}$ near $T_{c}$ as $m_{\pi}$ decreases from 200 to $135 \mathrm{MeV}$, similar to the scaling expected near an $O(4)$ or a number of other universal critical points, provided the regular part of $\chi_{\text {disc }}$ is small. However, in this region we find $\chi_{\text {disc }} 50 \%$ larger than that suggested by staggered fermion results, a discrepancy that will require further study to resolve.

These results represent an important milestone in the study of the QCD phase transition. The crossover character and pseudocritical temperature of the transition have now been obtained using a formulation which respects the symmetries of QCD, uses physical strange and light quark masses, and is performed at an inverse lattice spacing $1 / a \geq$ $1.1 \mathrm{GeV}$ where $5 \%$ discretization errors are expected. This is a challenging calculation with five-dimensional lattice volumes as large as $64^{3} \times 8 \times 24$ and a physically light quark mass. This study was only possible because of its use of the DSDR action [18], Möbius fermions [3], highly efficient code [19], and the petaflops-scale Sequoia and Vulcan computers at the Lawrence Livermore National Laboratory. Of course, it is important to explore these questions at larger spatial volume and smaller lattice spacing as adequate resources become available.
Computing support for this work on the LLNL Vulcan Blue Gene/Q supercomputer came from the Lawrence Livermore National Laboratory (LLNL) Institutional Computing Grand Challenge program. We gratefully acknowledge the high-performance Blue Gene/Q code created and provided by Peter Boyle and thank LLNL for funding from LDRD13-ERD-023. This work has been supported by the U. S. Department of Energy under Contract No. DE-AC52-07NA27344 (LLNL). N. H. C., Z. L., R.D. M., G. M., D. M., and H. Y. were supported in part by DOE Grant No. DE-FG02-92ER40699. T. B. and R. G. were supported in part by DOE Grant No. DE-KA1401020. H.-T. D., F. K., S. M., and P. P. were supported in part by DOE Grant No. DE-AC02-98CH10886. M. I. B. was supported in part by DOE Grant No. DE-FG0200ER41132. This work, and the development of software used in this work, has been supported in part through the Scientific Discovery through Advanced Computing (SciDAC) program funded by the U.S. Department of Energy, Office of Science, Advanced Scientific Computing Research and Nuclear Physics.

*nhc@phys.columbia.edu

[1] R. D. Pisarski and F. Wilczek, Phys. Rev. D 29, 338 (1984).

[2] A. Pelissetto and E. Vicari, Phys. Rev. D 88, 105018 (2013).

[3] R. C. Brower, H. Neff, and K. Orginos, arXiv:1206.5214.

[4] T. Blum et al., Phys. Rev. D 69, 074502 (2004).

[5] R. Arthur et al. (RBC Collaboration, UKQCD Collaboration), Phys. Rev. D 87, 094514 (2013).

[6] A. Bazavov et al. (HotQCD Collaboration), Phys. Rev. D 86, 094503 (2012).

[7] M. I. Buchoff et al., Phys. Rev. D 89, 054514 (2014).

[8] C. Schroeder (HotQCD), Proc. Sci., CPOD2013 (2013) 053.

[9] J. Engels, S. Holtmann, T. Mendes, and T. Schulze, Phys. Lett. B 514, 299 (2001).

[10] J. Engels and F. Karsch, Phys. Rev. D 85, 094506 (2012).

[11] J. Engels and F. Karsch, arXiv:1402.5302.

[12] J. Braun, B. Klein, and P. Piasecki, Eur. Phys. J. C 71, 1576 (2011).

[13] A. Bazavov et al., Phys. Rev. D 85, 054503 (2012).

[14] S. Borsányi, Z. Fodor, C. Hoelbling, S. D. Katz, S. Krieg, C. Ratti, and K. K. Szabó (Wuppertal-Budapest Collaboration), J. High Energy Phys. 09 (2010) 073.

[15] C. Bernard, T. Burch, C. DeTar, J. Osborn, S. Gottlieb, E. Gregory, D. Toussaint, U. Heller, and R. Sugar (MILC Collaboration), Phys. Rev. D 71, 034504 (2005).

[16] Y. Aoki, G. Endrodi, Z. Fodor, S. Katz, and K. Szabo, Nature (London) 443, 675 (2006).

[17] M. Cheng et al., Phys. Rev. D 74, 054507 (2006).

[18] D. Renfrew, T. Blum, N. Christ, R. Mawhinney, and P. Vranas, Proc. Sci., LATTICE2008 (2008) 048.

[19] P. Boyle, Proc. Sci., LATTICE2012 (2012) 020. 